\title{
ANALYSIS OF GENETIC DETERMINISM IN MICE INFECTED WITH Trypanosoma cruzi RESISTANT Y STRAIN
}

THESIS: L.A.C. Passos submitted this thesis for the degree of Doctor in Genetic (Immunology) publicly examined at the Institute of Biology, UNICAMP, Campinas, São Paulo, Brazil, 2003.

Advisor: Professor Júlia Keiko Sakurada

ABSTRACT: Chagas' disease is amongst the major health problems in South America, impairing the welfare of the population. Since its discovery, in 1909, by Carlos Chagas, American Trypanosomiasis showed significant differences in the resistance of infected people. Such observations led to the hypothesis that the host genetic background could have an influence on the development of this disease and on the lifespan of infected people. By the beginning of this century, the amount of publications aiming to investigate the genetic events and the regions of genome involved with the resistance to parasitic diseases has significantly increased. The strategies used in these studies follow similar protocols and are based on the use of informative crossings between non-related inbred strains, presenting different phenotypes on what refers to resistance or susceptibility to a certain disease, associated to the use of polymorphic molecular markers to track the frequency of specific alleles within the population. These strategies have allowed the identification of the genome regions involved in the resistance to experimental infection with different pathogens, such as $P$. chabaudi, $T$. congolensis, L. major T. gondii, and $M$. tuberculosis. In the specific case of $T$. cruzi, few experiments analyzing the genetic of resistance to experimental infection were performed in the early 80's and showed the importance of the $\mathrm{H} 2$ locus and possible involvement of other genes not related to this locus. However, there is no record in literature identifying the regions of mouse genome related to the resistance to experimental infection with $T$. cruzi. In the present work, informative crossings between $T$. cruzi resistant and susceptible strains have been made. The resistant second-generation offspring were analyzed and displayed important regions for the resistance against high doses of the parasites on chromosomes $7,11,14,17$, and 19. The identification of these regions allowed the development of a congenic recombinant animal model, an important tool to understand the involvement of the genes present in these regions in resistance to infection caused by $T$. cruzi.

KEY WORDS: Trypanosoma cruzi, genetic of resistance, congenic recombinant

CORRESPONDENCE TO: Luiz Augusto Corrêa Passos, Rua José Antônio Bruzon, 149, 13.190-000, Monte Mor, São Paulo, Brasil. Email: luizaugusto@cemib.unicamp.br 